\title{
Complex dynamics of passively mode-locked fiber lasers with strict polarization control
}

\author{
O. Pottiez ${ }^{a}$, Y. E. Bracamontes-Rodríguez ${ }^{a}$, J. P. Lauterio-Cruz ${ }^{a, b}$, H. E. Ibarra-Villalón ${ }^{a, c}$, J. C. Hernández-García ${ }^{b, d}$, \\ H. Santiago-Hernández ${ }^{a}$, E. García-Sánchez ${ }^{a}$, O.S. Torrés-Muñóz ${ }^{a}$, and M. Bello-Jiménez ${ }^{e}$ \\ ${ }^{a}$ Centro de Investigaciones en Óptica, \\ A. C., Lomas del Bosque 115, Col. Lomas del Campestre, León, Gto. 37150, México. \\ ${ }^{b}$ Departamento de Electrónica, División de Ingenierías CIS, Universidad de Guanajuato, \\ Carretera Salamanca-Valle de Santiago Km $3.5+1.8 \mathrm{Km}$, Comunidad de Palo Blanco, Salamanca, Gto. 36885, México. \\ ${ }^{c}$ Departamento de Ciencias Básicas, Universidad Autónoma Metropolitana-Unidad Azcapotzalco, \\ Av. San Pablo No. 180. Col. Reynosa Tamaulipas, Azcapotzalco, CDMX. 02200 México. \\ ${ }^{d}$ Consejo Nacional de Ciencia y Tecnología, \\ Av. Insurgentes Sur No. 1582, Col. Crédito Constructor, Del. Benito Juárez, C.P. 039040, México. \\ ${ }^{e}$ Instituto de investigación en Comunicación Óptica, \\ Universidad Autónoma de San Luis Potosí, Av. Karakorum No. 1470 Lomas 4aSecc., 78210 San Luis Potosí, México.
}

Received 5 October 2020; accepted 26 October 2020

\begin{abstract}
In this work, we browse an overview of the complex dynamics that arise in the non-stationary modes of operation of partly mode-locked fiber lasers under strict polarization control. Both ring and figure-eight cavities implementing saturable absorber mechanisms that rely on nonlinear polarization rotation were considered. The use of twisted fiber ensures robust operation, whereas precise polarization adjustments allow tailoring the nonlinear switching characteristic in a clear and repeatable manner. The enhanced flexibility of these sources allowed identifying and studying a broad spectrum of peculiar dynamics, in particular by implementing the temporal and spectral mapping techniques. These include the emergence, fusion, splitting, complex evolution, and eventual decay of chaotic bunches of radiation, which are also subject to large spectral shifts, ample quasi-periodic instabilities, extreme-intensity events or the creation of solitons, among others, as well as the complex evolution of massively multiple soliton states. Finally, with the help of numerical simulation, several aspects of the physics underlying these puzzling behaviors could be elucidated.
\end{abstract}

Keywords: Fiber lasers; passive mode locking; noise-like pulses; soliton dynamics; optical rogue waves.

PACS: 42.55.Wd; 42.65.Re

\section{Introduction}

Passively mode-locked fiber lasers are versatile and low-cost sources of many categories of optical pulses that are attractive for applications. In these lasers, pulse formation relies on the use of a saturable absorber (SA). Aside from schemes that employ physical SAs (for example, semiconductor SA mirror or SESAM, carbon nanotubes, or graphene-based SAs), the two most common schemes are the ring and the figure-eight cavities exploiting the Kerr effect in fiber to provide artificial SA action [1,2]. In these sources, in particular, if conventional (non-polarization-maintaining) fiber is used, polarization adjustments (realized in practice through polarization controllers) are important as they allow biasing the nonlinear characteristic to ensure SA operation (transmission increasing with increasing power), as opposed to intensity-limiting action (transmission decreasing with power), which does not favor mode-locking. However, because polarization evolves in a random, uncontrolled way in conventional fibers, such procedure is usually empirical, settings are hardly repeatable and are subject to frequent readjustments due to the environmental dependence of the fiber residual birefringence. Besides, the mode of operation of the laser and the pulse properties do not depend only on the sign of the slope of the non- linear characteristic but also on the detailed transmission parameters (switching power, dynamic range, etc.), which can hardly be controlled through such blind adjustment procedure.

A significant improvement in the control of the operation of passively mode-locked fiber laser schemes was made possible through the inclusion of twisted fiber in their design. Twisted fiber essentially behaves in a way similar to an ideal isotropic fiber [3]. Indeed, twist induces precession of the fiber birefringence axes, which tends to eliminate the fiber residual birefringence [4]. Hence, given some input polarization ellipticity (linear, circular, or in general elliptic, which can be set using a wave retarder plate, for example), this ellipticity will be preserved during propagation. Knowing and controlling the polarization state at all positions along the fiber (and not only at the output of a polarizer or polarization controller) is important to determine the artificial SA action in passively mode-locked fiber laser schemes because the Kerr effect is polarization-dependent, being maximal and minimal for linear and circular polarizations, respectively, for a given value of power [5]. It should be noted finally that, whereas twisting efficiently eliminates the fiber residual birefringence, ensuring the preservation of the polarization ellipse, it also introduces some amount of circular 
birefringence (optical activity) so that the ellipse azimuth is not preserved and rotates during propagation. This rotation however only introduces a fixed bias that does not affect, essentially, the SA action. In particular, the Kerr effect, which in the first approximation only affects polarization through a power-dependent rotation of the ellipse (nonlinear polarization rotation, NPR) in twisted fibers [6], only depends on ellipticity, not on polarization azimuth.

The extensive study of twisted fiber properties and its use in the design of enhanced fiber devices and lasers was carried out primarily by E. A. Kuzin and coworkers; regrettably, its significance is often underrated among the scientific community. In a smart experiment, these authors demonstrated that even with relatively moderate twist rates ( 5-6 turns/m), ellipticity is fairly well preserved even after propagation through a quarter of a kilometer of standard fiber, provided that the spool diameter is large enough to avoid curvature-induced linear birefringence [7]. This result is particularly impressive if one considers that it typically takes only a few meters of propagation or less through standard fiber to get the polarization state significantly altered if no special precaution is taken. This property allowed the design of robust devices based on twisted fibers, among which the power-symmetric, polarization-imbalanced nonlinear optical loop mirror (PI-NOLM) occupies a particular position [6]. With this device, precise polarization control allows adjusting not only the bias but also the slope, switching power, and dynamic range (contrast) of the nonlinear switching characteristic [8-11]. More elaborate schemes based on the PINOLM design also allowed to further enhance the flexibility of the nonlinear switching characteristic, yielding sharp, flattopped and even square-like transmission curves. [12-14]. The flexibility and robustness of such NOLM-based devices with strict polarization control allowed envisioning their use in optical transmission and data processing systems to perform a wide range of essential operations including pedestal removal, all-optical signal regeneration [15-19], even at various wavelengths simultaneously for WDM systems [20] or in the case of complex, multilevel modulation formats (constellations) [21], wavelength conversion for OTDM systems [22], as well as all-optical logic gates [14]. Other noticeable uses of the PI-NOLM include the determination of the temporal profile of ultrashort optical pulses [23], as well as estimating the amplitude statistics of a bunch of optical pulses $[24,25]$, which provided in particular one of the first experimental pieces of evidence of the emergence of ultrashort extreme events (optical rogue waves, ORWs) in noise-like pulses (NLPs) [25].

Finally, the PI-NOLM with twisted fiber was used as $\mathrm{SA}$ in the design of passively mode-locked figure-eight laser schemes [26]. Strict polarization control allows precise control of the switching characteristic, which in turn allows selecting the regime of operation and type of generated pulses, and also allows adjusting the pulse properties over a wide range through well-defined wave retarder adjustments $[27,28]$. In addition to figure-eight lasers, ring-cavity fiber lasers consisting of twisted fiber were also designed [29,30]. In this case, the mode-locking mechanism is provided by NPR taking place in the twisted fiber ring together with a polarizer inserted in the cavity. Similar to figure-eight schemes, including a PI-NOLM, strict polarization control in ring cavities allows adjusting the bias, slope, switching power, and dynamic range of the switching characteristic. Such schemes made it possible to relate each setting of the polarization state (ellipticity and azimuth) to a specific mode of operation of the laser (polarization mapping) [29]. In one setup, precise polarization adjustments allowed selecting among as much as 10 different regimes, which encompass single- or dualwavelength operation, solitons, NLPs, or hybrid soliton-NLP emission, in a well-defined and repeatable way [30]. Such results, besides shading light on the mechanisms and specific conditions leading to the onset of each particular pulsed regime in these sources, are also crucial in making them attractive for potential applications, where mode-locked fiber sources have been traditionally discarded due to their difficult control and environmental sensitivity.

Beyond stable mode-locking operation, where the pulses repeat almost identically at each round-trip, passively modelocked fiber lasers can display a broad range of nonstationary regimes, in which pulse properties vary dynamically across the cycles. First discarded by the scientific community, these unconventional regimes have attracted considerable attention throughout the last decade. Among those regimes, one finds, for example, various types of pulsating instabilities [31], soliton explosions [32], multiple solitons dynamics [33,34], and noise-like pulsing [35-43]. A frequently related subject is the quest for extreme-intensity optical events (ORWs) [25,3945]. However, given the extreme diversity and richness of those complex regimes, most of which are still puzzling researchers, the subject can hardly be reduced to a few keywords. The complexity of these regimes and the difficulty of their understanding arises from the large number of physical phenomena that are involved in their emergence, some of which are very subtle and difficult to control; such is the case of polarization. In this context, strict control of the polarization state in the cavity is crucial to precisely define the conditions under which each specific dynamic arises, in a repeatable way, allowing for their proper study. In this paper, we browse a general overview of the research activities of our group over the last 5 years, in the frame of nonstationary operation of mode-locked fiber sources with strict polarization control. The work covers the two most common architectures of passively mode-locked fiber lasers, namely the ring and figure-eight configurations. Other configurations are possible; however, such as the Fabry-Perot configuration, which would introduce some differences in the laser operation and its modeling, in particular, related to the double pass per round-trip through elements such as the saturable absorber and gain medium. We believe, however, that behaviors similar to those described here can be expected in such sources as well, as these regimes in general do not depend on the general cavity configuration but instead on parame- 
ters such as cavity length, dispersion map, saturable absorber characteristics, birefringence and gain medium, among the most important.

\section{Experimental setups and principles of oper- ation}

The experimental schemes used to produce different kinds of pulses and dynamics in this work are presented in Fig. 1. These two schemes were used, with little variations, to produce all the experimental data reported here. Figure 1a) is a twisted ring laser design in which NPR is used in combination with a polarizer to provide a saturable absorption mechanism. In such a scheme, the SA transmission writes as:

$$
T=\frac{1}{2}+\frac{\sqrt{1-A c^{2}}}{2} \cos \left(2\left[\psi-\alpha_{p}+\frac{1}{3} A_{c} \gamma P L\right]\right),
$$

where $A_{c}$ is the first Stokes parameter (related to ellipticity), $\psi$ is the ellipse azimuth, $\alpha_{P}$ is the polarizer angle and $\gamma, P$ and $L$ are the Kerr nonlinear coefficient $(=1.5 / \mathrm{W} / \mathrm{km}$ in standard fiber for linear polarization), optical power and fiber length (=20 m in this case), respectively. For the sake of simplicity, the fixed bias introduced by circular birefringence of the twisted fiber was not accounted for in Eq. (1). In the scheme of Fig. 1a), the polarization state is precisely adjusted by a quarter-wave retarder (QWR), and a variable wave retarder (VWR) inserted just after the polarizer. The VWR is a very convenient device to control separately the ellipticity and azimuth, through pressure and rotation, respectively [30]. This procedure to adjust the polarization state $\left(A_{c}\right.$ and $\psi$ in Eq. (1)) in turn allows controlling the nonlinear switching characteristic (switching power, dynamic range, etc.) in a precise and repeatable way, to favor the emergence of a given operational regime $[29,30]$.

On the other hand, Fig. 1(b) presents the figure-eight laser scheme, which includes a polarization-imbalanced NOLM as the SA [6]. This type of NOLM includes a 50/50 coupler (so that this scheme is power-symmetric, contrary to other, more common NOLM designs), $100 \mathrm{~m}$ of twisted standard fiber, and a QWR plate that breaks the symmetry between the counterpropagating beams in the loop. In this scheme, switching thus relies not on power imbalance but on the different ellipticities of the counter-propagating beams, which causes different amounts of NPR to accumulate through the loop. Depending on polarization adjustments (polarization state at the NOLM input and QWR orientation), a broad variety of transmission characteristics can be generated [8]. In Fig. 1(b), a polarizer and half-wave retarder (HWR) are inserted at the NOLM input so that linear polarization with adjustable azimuth is introduced to the NOLM input. In this case, the transmission is given by [8]:

$$
\begin{aligned}
T & =\frac{1}{2}-\frac{1}{2} \cos (2 \alpha) \\
& \times\left(\frac{1}{6} \gamma L_{N} \sin [2\{\alpha+\psi\}] P_{i n}-2 \alpha\right)
\end{aligned}
$$
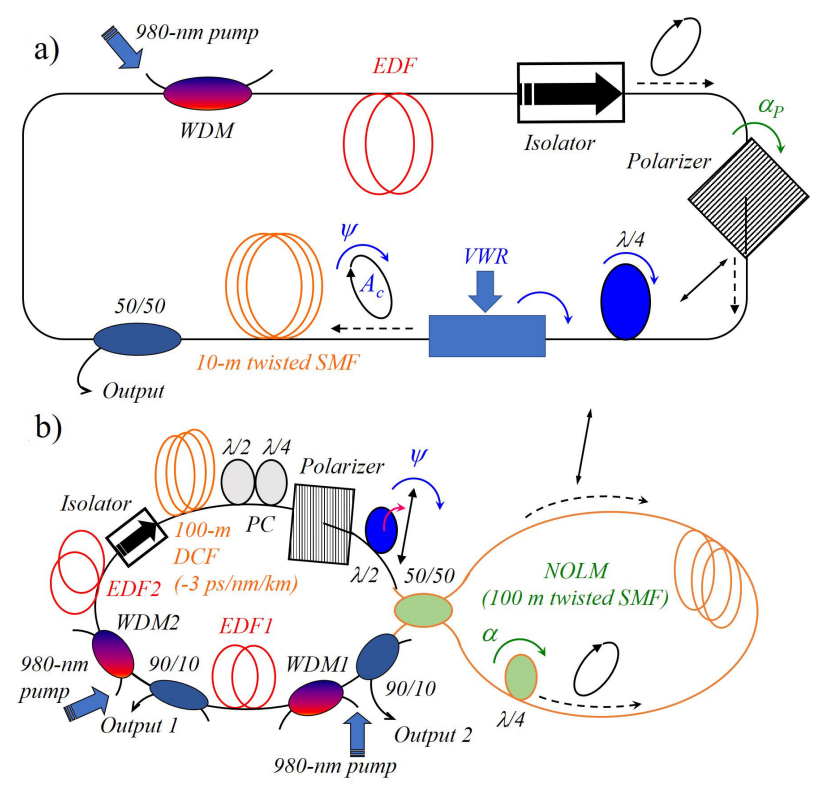

FIGURE 1. Fiber laser-schemes used in this work. (a) $20 \mathrm{~m}$ ring cavity; (b) $220 \mathrm{~m}$ figure-eight cavity. Both cavities have anomalous average dispersion. $980 \mathrm{~nm}$ pump powers ranging from $\sim 100$ to $\sim 300 \mathrm{~mW}$ were used for the results presented in this work. EDF: erbium-doped fiber; WDM: wavelength division multiplexing coupler; SMF: standard single-mode fiber (dispersion $\sim 17 \mathrm{ps} / \mathrm{nm} / \mathrm{km}$ ); DCF: dispersion compensating fiber; VWR: variable wave retarder; PC: polarization controller.

where $L_{N}$ is the loop length, $P_{i n}$ is the NOLM input power, and $\alpha$ and $\gamma$ are the QWR angle and input polarization azimuth, respectively (again, the bias angle introduced by circular birefringence was not accounted for). Hence, controlling QWR and input polarization angles (the latter through the HWR) allows controlling the switching power, dynamic range, and bias of the switching characteristic. This flexibility again was crucial for adjusting the mode of operation of the laser.

\section{Experimental results and discussions}

\subsection{Multiple NLP operation}

Beyond soliton operation, a common regime of passively mode-locked fiber lasers is NLP generation [35-43]. An NLP is a large $(\sim n s)$ bunch of radiation, which contains a fine inner structure at sub-ps scale. Although in many cases its average envelope remains roughly stable over time, the internal sub-ps spikes are evolving and interacting constantly, in a chaotic way. NLPs are attracting attention for applications, such as micromachining, in particular, because they can reach very high energies without breaking. Still, in some cases, as pump power is increased or birefringence adjustments are performed, a single NLP tends to break up into multiple smaller, roughly identical sub-pulses [46-48], a phenomenon that until recently was not clearly understood. In 


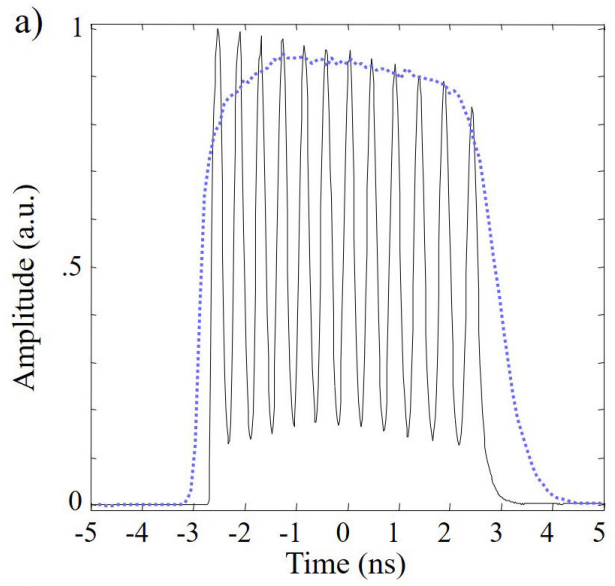

c)

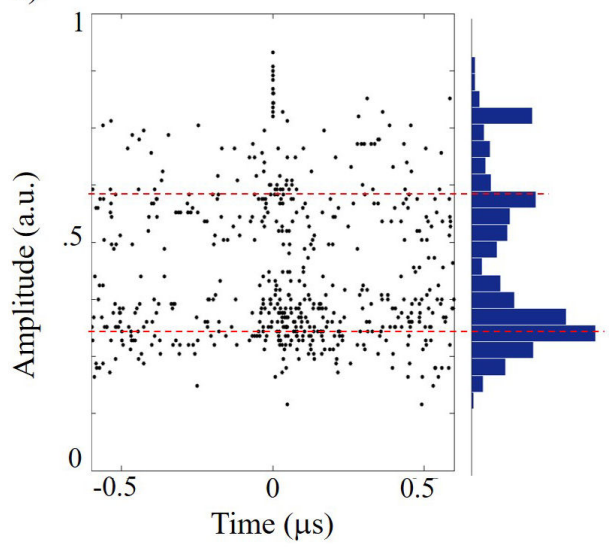

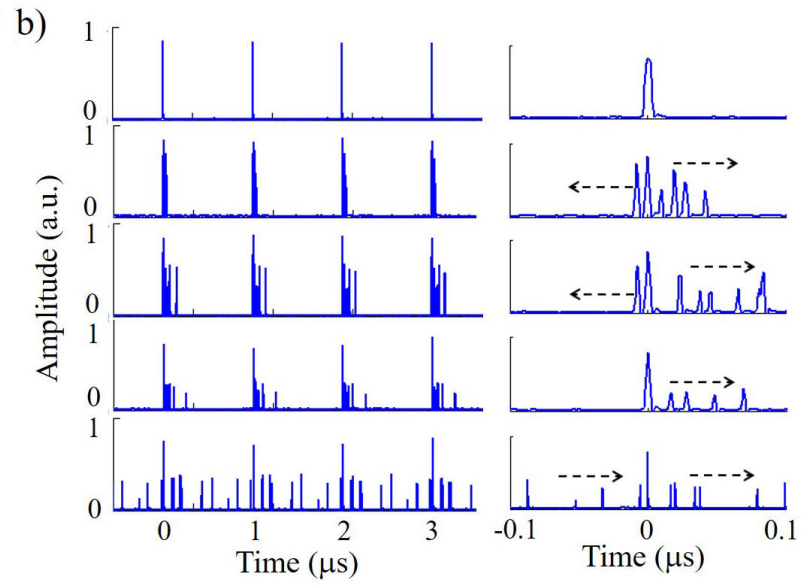

d)
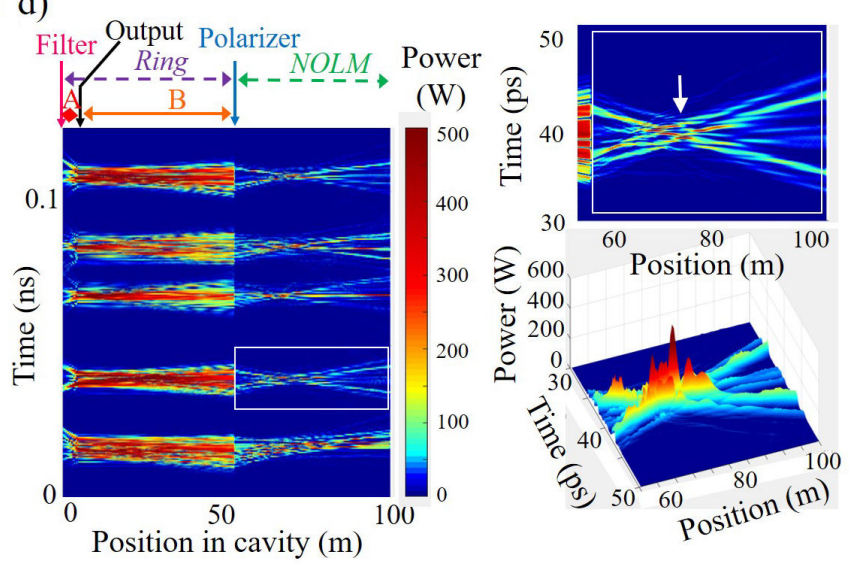

FIGURE 2. a) Single NLP with rectangular (dashed line) and comb-like (solid) profile, observed on a $25 \mathrm{GHz}$ sampling scope. b) $200 \mathrm{MHz}$ scope traces showing different degrees of NLP splitting for slightly different retarder adjustments (left: single-shot measurements over $\sim 4$ periods; right: close-ups). c) Energy statistics of the sub-pulses in (b). d) Multiple NLP evolution over one cavity round-trip (numerical); ring section includes amplifier section A (dispersion $=-70 \mathrm{ps} / \mathrm{nm} / \mathrm{km})$ and passive fiber B $(-5 \mathrm{ps} / \mathrm{nm} / \mathrm{km}) ; \mathrm{NOLM}$ section: $15 \mathrm{ps} / \mathrm{nm} / \mathrm{km}$. Right: close-up views of the evolution inside the NOLM (clockwise direction) for one of the bunches (white box).

some cases, a very large number of NLPs, up to thousands, was observed in the cavity $[49,50]$.

Depending on the adjustments of the wave retarders, the figure-eight laser scheme (Fig. 1b)) could be operated in either single- or multiple-NLP operation [47]. In most cases, a single NLP has the shape of a long rectangle; in some cases; however, a comb-like envelope was observed (Fig. 2a)), revealing a series of similar, uniformly spaced sub-ns structures, whose number is variable (from 4 up to 17 sub-pulses were observed in the same bunch) [47,51]. These comblike profiles prefigure the splitting of the NLP into multiple sub-packets. Figure $2 b$ ) shows that, through subtle successive wave retarder adjustments, it was possible to observe the evolution from single-NLP operation to multiple pulsing, passing through different degrees of pulse splitting, as the main bunch progressively loses cohesion and disintegrates into an increasing number of sub-units, which move further and further away from the main bunch [52]. This series of single-shot scope measurements, obtained for slightly different wave retarder adjustments, suggest that in each case, a dynamic evolution takes place (in particular, the sub-pulses move away from the main bunch). This was confirmed visually by direct observation of the oscilloscope in continuous run mode; however, the temporal sequence of events was not registered at that stage. Instead, we performed a statistical study of the energies of the sub-packets (Fig. 2c)). This analysis showed that these energies take preferentially discrete values, integer multiples of a fundamental quantity. We concluded that the sub-pulses have roughly the same energy, some sub-packets containing two or more closely spaced units, which could not be discriminated by the $200 \mathrm{MHz}$ oscilloscope.

Those experimental findings were partially confirmed by numerical simulations, where propagation in the fiber cavity was modeled through the generalized Schrödinger equation [53]. As a result, the formation of multiple pulses having roughly the same duration and energy, instead of one large, single NLP, was observed. We attributed the phenomenon to dispersion management in the cavity, where fiber sections with values of dispersion are large but of opposite signs (i.e., normal and anomalous) alternate in the cavity. Simply stated, in a dispersion-managed cavity, small (sub-ns) pulses un- 

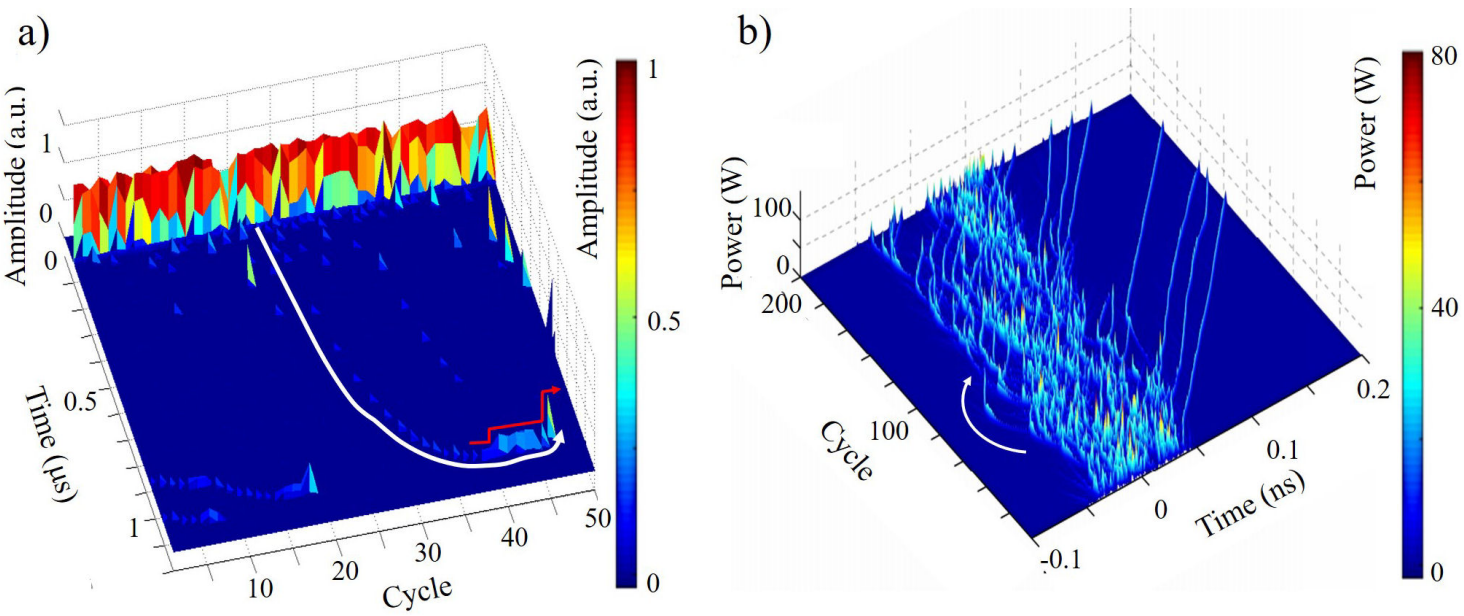

FIGURE 3. a) Temporal sequence showing an intense NLP and drifting smaller sub-packets. b) Simulation results (one curved trajectory bearing some similarity with the one of a) is highlighted).

dergo significant reshaping along the cavity (Fig. 2d)); in particular, in the NOLM section, the pulses are significantly compressed, meaning that peak power increases (close-up views in Fig. 2d)). In contrast, if one assumes that a single, long (several ns) NLP circulates in the cavity, variations of its envelope over one cycle under the action of dispersion would be far less significant, in particular, its intensity would not grow significantly in the NOLM section. As a consequence, multiple small NLPs, instead of a long single bunch, tend to form in the cavity because higher intensity means higher transmission through the NOLM, and thus lower cavity losses, in the former case. Although it was exemplified here in the case of a figure-eight cavity, the same principle applies for dispersion-managed ring lasers. These results also emphasize the importance of taking into account the details of the cavity construction, and not only average quantities (for example, average dispersion), as some numerical models do (in particular, the Ginzburg-Landau equation), in order to be able to describe and reproduce accurately this kind of complex behaviors of fiber lasers.

\subsection{Complex NLP dynamics}

A much clearer idea of the dynamics taking place in the laser can be obtained by the temporal mapping technique [36-38,41]. An example is illustrated in Fig. 3a) [54]. A series of single-shot measurements with $\sim 2250 \mathrm{~ms}$ spacing (which corresponds to $\sim 250,000$ cavity cycles) were performed using a computer-controlled $200 \mathrm{MHz}$ oscilloscope. These measurements were then stacked, yielding a 3-D (or color-scale) representation of the pulses evolution. The figure has two-time axes: one fast time (the time of each scope acquisition) and one slow time (showing the evolution over the cycles, here covering several seconds).

Figure 3a) shows the main NLP in the process of liberating sub-packets, which then drift away from the main bunch, describe complex trajectories, and eventually vanish. A particular trajectory that could be captured completely (from its emergence out of the main bunch to its eventual decay) over the measurement time is highlighted by a white line: after drifting away at nearly constant speed, it slows down, remains stationary for a while, and even retrocedes slightly, before vanishing. It has to be noted that the amplitude of the sub-packet varies, in discrete steps, during this process (red arrow in Fig. 3a)). Remarkably, the amplitude does not decay but instead grows progressively in a staircase-like manner, and the sub-packet abruptly vanishes just after reaching its maximal amplitude. Portions of the trajectories of other sub-packets are also visible in Fig. 3a), which do not necessarily follow the same evolution. These results can be paralleled with those of the previous section; however, much more information is provided in this case, as the sequence of the temporal evolution is recorded, which allows gaining much deeper insight into the dynamics than conventional statistical analysis.

A broad range of complex dynamics and processes were evidenced, including the emergence, splitting, fusion of multiple sub-packets that follow diverse evolution patterns before eventually decaying, over time scales that range from a few seconds to half an hour [54,55]. Some of the complex behaviors evidenced in this work were recently reproduced numerically (Fig. 3(b)) [56], although over very different time scales (numerical results show the evolution over successive cycles, instead of every $250 \mathrm{~ms}$ ). In particular, the drift of sub-packets relative to the main bunch and variations of trajectory over time can be attributed to changes of the central wavelength of the packets in the dispersive cavity, and possibly also to dissipative effects, such as a temporal gradient of nonlinear losses through the NOLM.

Finally, another kind of complex dynamics, involving quasi-periodic energy fluctuations, wavelength shifts, extreme-intensity events, the formation of solitons in a NLP, and even NLP to solitons conversion, was observed experimentally and described in a ring laser cavity (Fig. 1a)) $[57,58]$. In this case, measurements were performed using 

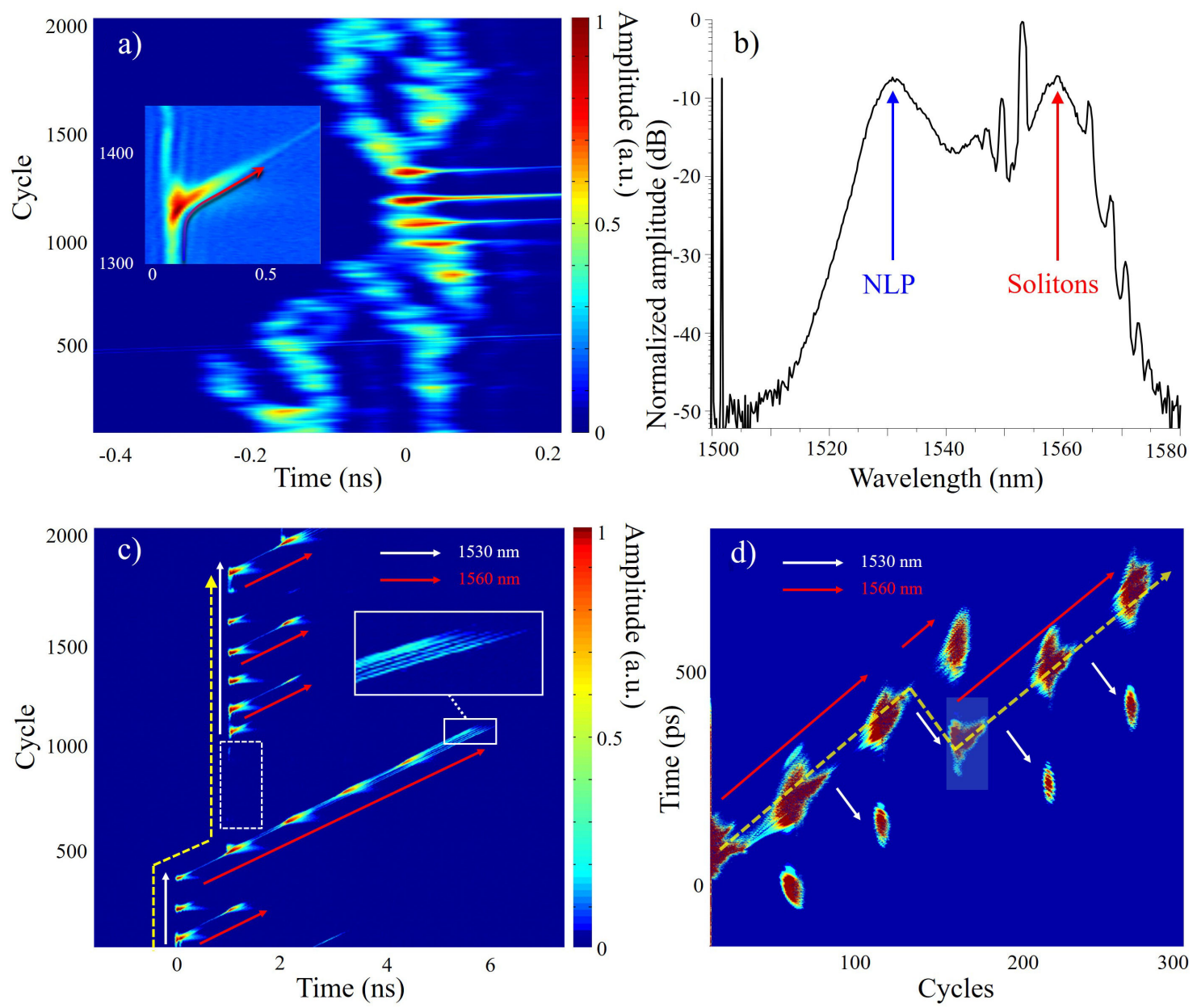

FIGURE 4. a) Temporal mapping of complex NLP evolution, including extreme-intensity events (red spots) and creation of solitons (diagonal lines). b) Averaged spectrum measured with an optical signal analyzer (OSA). c) Quasi-periodic oscillatory regime (close-up view: solitons). d) Simulation results using a model that includes wavelength-dependent gain saturation dynamics.

a $16 \mathrm{GHz}$ fast oscilloscope. Long, single-shot measurements covering a few thousands of cycles were performed; the data was then sliced into single-period sections, which were then stacked to obtain the 3-D mapping. Figure 4(a) shows an example of complex NLP evolution, where the broad temporal waveform occasionally focuses into intense, narrow spots that qualify as ORWs. The thin diagonal lines that emerge from these spots are the trajectories of solitons, which are created inside the NLP during the high-intensity events. The inclination of these trajectories (as opposed to the NLP, which follows a roughly vertical direction) denotes that they have a different wavelength and thus walk off from the NLP in the dispersive cavity. Indeed, whereas the NLP has a spectrum centered in the $1530 \mathrm{~nm}$ region, the solitons appear in the $1560 \mathrm{~nm}$ region (Fig. 4(b)). Hence, during an extreme event, the NLP not only produces a soliton, but the latter is also red-shifted by $\sim 30 \mathrm{~nm}$ in the process. The inset in Fig. 4a) provides a close-up view of one of the events, showing the creation of a soliton and progressive deflection of its trajectory, which corresponds to its gradual red-shift.

Through slight wave retarder adjustments, the occasional intense events taking place in the NLP evolution as described above tend to repeat in an almost periodic fashion, yielding a quasi-periodic oscillatory behavior. Such a regime is illustrated in Fig. 4c). The red spots show the NLP at the maxima of oscillations; again, vertical trajectories correspond to $1530 \mathrm{~nm}$ components, whereas the diagonal trajectories represent $1560 \mathrm{~nm}$ radiation. Hence, the figure shows that the oscillations of the overall dominant $1530 \mathrm{~nm}$ component (see yellow dashed arrow, mainly vertical trajectory) is accompanied by the quasi-periodic conversion of part of the radiation to the $1560 \mathrm{~nm}$ region, which walks off the main bunch (diagonal trajectories). Most of this red-shifted radiation decays after the next maximum of oscillation; in one case, however, the diagonal trajectory extends much further until the NLP evolves into a packet of solitons (see close-up in Fig. 4c)), which eventually decay. It is also noticeable that, over the duration of this long trajectory (cycles $\sim 500$ to $\sim 1000$ ), the $1560 \mathrm{~nm}$ component transitorily becomes dominant (whereas the $1530 \mathrm{~nm}$ component almost disappears, see the white dashed 

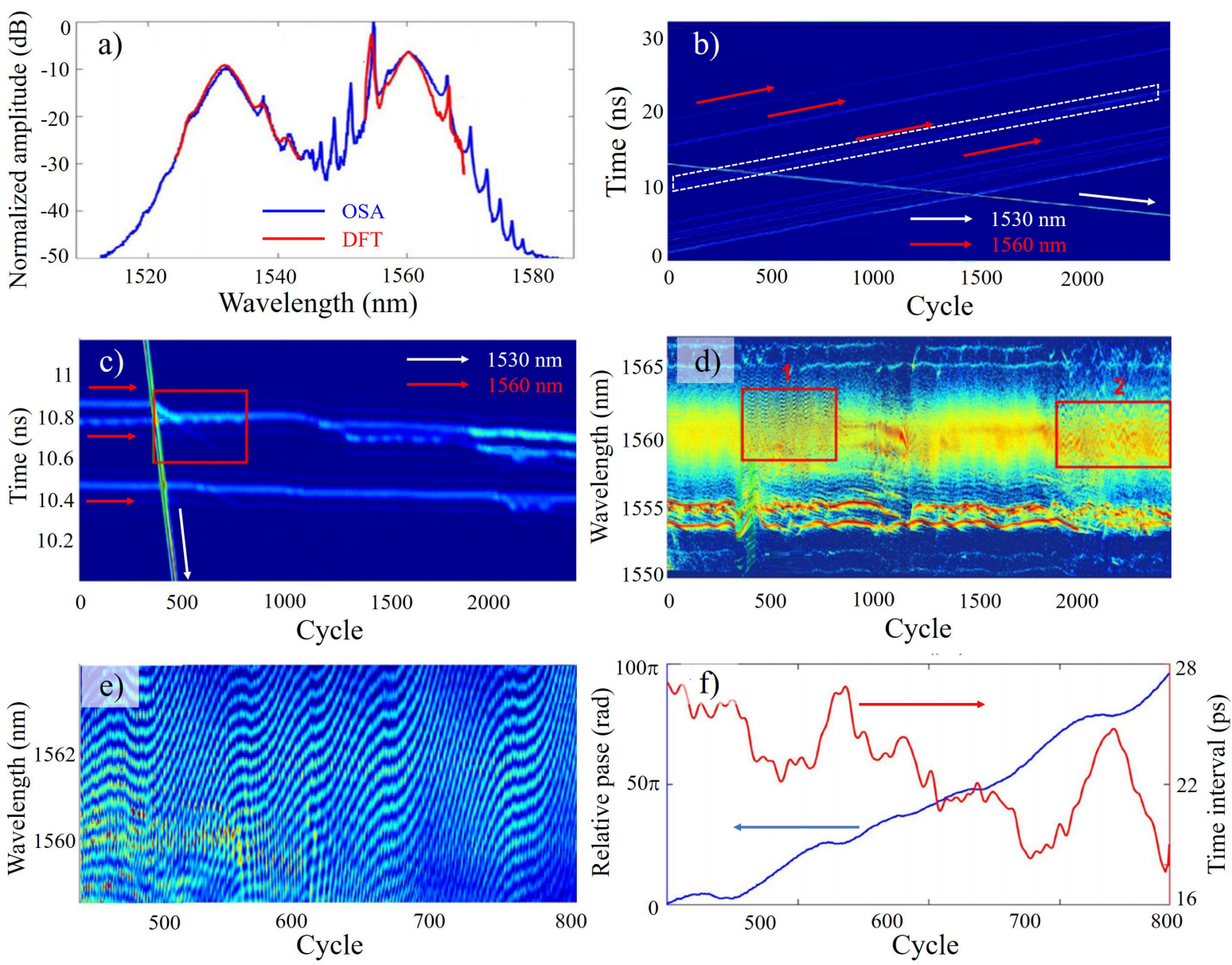

FIGURE 5. a) Average spectrum of multiple soliton dynamics (DFT curves were obtained by averaging single-shot spectra separately for each wavelength component). b) Complete temporal mapping. c) Detail of one 1560-nm packet. d) Corresponding spectral evolution (red boxes highlight interference patterns). e) Detailed view of the first fringe pattern (box 1 in d)). f) Pulse separation and relative phase determined from e).

box), after which it vanishes and the $1530 \mathrm{~nm}$ component regains supremacy. Although the vertical trajectory is eventually restored, it is shifted by $\sim 1 \mathrm{~ns}$ to the right, as a result of a double wavelength conversion process ( $1530 \mathrm{~nm}$ to 1560 $\mathrm{nm}$, then back to $1530 \mathrm{~nm}$, yellow dashed arrow).

Complex dynamics as the one illustrated in Fig. 4c), as well as related dynamics reported by several authors [37,41,59], which include in particular intense events, quasiperiodic oscillations and sudden kinks in the trajectories (attributable to wavelength shifts) can be explained by gain dynamics. We developed a numerical model that takes into account gain dynamics, in particular, the strong wavelength dependence of gain saturation in erbium fibers [60]. In other words, in this model, the shape of the gain spectrum varies significantly depending on the saturation level: whereas the small-signal (unsaturated) gain peaks at 1530 $\mathrm{nm}$, the strongly saturated gain presents a maximum in the $1550 \mathrm{~nm}$ region. As illustrated in Fig. 4d), this model allowed reproducing numerically and understanding this kind of dynamics, including aspects such as the emergence of intense events, quasi-periodic energy oscillations, wavelength shifting, walk-off and decay of portions of the bunch of radiation, and even temporal displacement of the main radiation component as a result of a double wavelength conversion process (yellow dashed arrow). Again, an approach based on the analysis of temporal sequences, both in the experiment and numerical simulations, is very useful to shed light on the processes involved in the observed phenomena. This is particularly true for the understanding of extreme-intensity events (ORWs), which have been traditionally defined and studied by statistical means (histograms) [25,44].

\subsection{Multiple soliton dynamics}

Fast scope measurements can also be used to study the dynamics in the spectral domain (spectral mapping, also called real-time spectroscopy). Single-shot optical spectra can be obtained from the time-domain signal through the dispersive Fourier transform (DFT), which in practice simply consists of using a long dispersive fiber before detection: the pulse is stretched temporally until its shape no longer relates to the original temporal waveform, but instead is an image of its 
optical spectrum [39,40,43,45]. Such measurements allow building the sequence of the single-shot spectra over successive round-trips and analyzing their evolution, in much the same way as in the time domain. Spectral mapping thus gives much more information on the optical spectrum and its realtime evolution than a measurement from an optical signal analyzer (OSA), which only provides a single, averaged spectrum.

As the fast oscilloscope includes multiple input channels, it is possible to perform both temporal and DFT measurements at the same time. We applied both temporal and spectral mapping techniques simultaneously to study several aspects of the NLP dynamics in the figure-eight laser (Fig. 1b)), including the splitting of an NLP into multiple sub-pulses and their merging in connection with quasi-periodic energy fluctuations, and also evidenced subtle wavelength shifts between the two laser outputs, which are attributable to gain dynamics [61].

The two techniques were also applied simultaneously to the characterization of a dual-wavelength, massively multiple soliton dynamics in the ring laser (Fig. 1a)) [62]. The optical spectrum (Fig. 5a)) indicates that solitons (whose spectra display the typical Kelly sidebands) are present at two wavelengths, $1530 \mathrm{~nm}$, and $1560 \mathrm{~nm}$. This is confirmed by examining the general time-domain sequence (Fig. 5b)), which shows that most soliton packets at $1560 \mathrm{~nm}$ are crossed by a single packet at $1530 \mathrm{~nm}$. Most trajectories are not single solitons, but actually, packets of several solitons, as will be confirmed in the following. As a small sample of this very complex dynamics, which cannot be fully described in detail here, we consider Fig. 5c), which shows a close-up view of the evolution of one of these $1560 \mathrm{~nm}$ packets (white box in Fig. 5b)). During the initial cycles, on the left of Fig. 5(c), this $1560 \mathrm{~nm}$ component consists of three trajectories, which are likely three individual solitons; however, after the collision with the $1530 \mathrm{~nm}$ packet (diagonal trajectory), two of them are brought close together, and seem to merge into one single trajectory (red box). However, this contradicted by the corresponding spectral map (Fig. 5d)) which shows the appearance of a periodic modulation of the spectrum (red box 1), meaning that two solitons are still present (another such interference pattern is found later in the sequence, red box 2 , which will not be discussed here). This fringe pattern (enlarged in Fig. 5(e)) actually contains detailed information on this pulse pair, allowing to calculate both their separation and relative phase, and their evolution during the cycles (Fig. 5(f)) [61]. Their separation is found to be smaller than $30 \mathrm{ps}$ at all times, below the resolution limit of the oscilloscope (60 ps), which explains that they cannot be resolved in the time domain. Hence, thanks to the DFT, it is possible to infer information that is not accessible in the time domain due to equipment limitations. The application of both time-domain and spectral mapping simultaneously is thus very fruitful, providing more complete and detailed information on the dynamics than each of these techniques taken separately.

Figure 5f) shows that the pulse separation evolves chaot- ically along with the cycles, although it remains confined within a relatively narrow range between $\sim 18$ and 28 ps. As to the relative phase, it grows in steps, with alternating stages of fast growth and of relative steadiness, or even slight decrease. However, the extension of these stages is not uniform across the sequence. Overall, the dynamics of this pulse pair (and other soliton molecules analyzed in this study) are more chaotic and far more irregular than the ones described in other references using the DFT technique $[63,64]$, possibly due to the longer cavity length.

\section{Conclusions}

In this paper, we reviewed several of the most complex dynamics that were recently evidenced in passively modelocked fiber lasers operating under incomplete mode-locking conditions. Both a twisted-fiber ring cavity and a figure-eight laser based on a twisted, polarization-imbalanced NOLM were analyzed. A broad spectrum of nonstationary regimes could be accessed, and precise setting and even fine tuning of each of them was made possible through strict polarization control implemented in these sources. The temporal and spectral mapping techniques were used, in some cases simultaneously, to precisely characterize each mode of operation. The NLP regime is a particularly fertile ground to evidence such behaviors. In some cases, a long single NLP tends to disintegrate into multiple sub-packets having similar energies and durations. This tendency is intimately related to the details of the cavity construction, in particular, dispersion mapping has a determinant role in favoring multiple NLP operation, as it was confirmed through numerical simulations. On the other hand, the complex evolutions and trajectories of these multiple packets are the result of subtle effects, such as temporal gradients of gain and loss. Besides, exotic effects, including extreme-intensity events, quasi-periodic instabilities, sudden wavelength shifts, and the emergence of solitons were also found to play a part in the NLP evolution; such scenarios could be explained and reproduced numerically in terms of wavelength-dependent gain dynamics. Finally, a dual-wavelength, strongly multiple soliton regime was analyzed, which allowed in particular to study the effect of collisions between different soliton packets or to probe in a very precise manner the complex evolution during the cycles of both the relative phase and separation between closely spaced solitons in each packet. We believe that this broad collection of results constitutes a significant step forward towards a clear description of several complex dynamics arising in these sources and that it also contributed to shed light on the physical processes involved in such behaviors, which were previously intriguing researchers. Finally, we hope that these advances will be useful to improve our understanding and control over the multiple modes of operation of these fiber laser sources, which in turn will make them attractive for applications even well beyond the realm of stable mode-locking regimes. 


\section{Acknowledgments}

This work was supported mainly by CONACyT grants CB 130966, CF 471 and CB 253925.

1. V. J. Matsas, D. J. Richardson, T. P. Newson, and D. N. Payne, Opt. Lett. 18 (1993) 358. https://doi.org/10.1364/ OL.18.000358

2. I.N. Duling III, Opt. Lett. 16 (1991) 539. https://doi. org/10.1364/OL.16.000539

3. T. Tanemura and K. Kikuchi, J. Lightwave Technol. 24 (2006) 4108.

4. S.F. Feldman, D.A. Weinberger, and H.G. Winful, J. Opt. Soc. Am. B 10 (1993) 1191. https://doi.org/10.1364/ JOSAB.10.001191

5. G. Agrawal, Nonlinear Fiber Optics, 6th ed. (Academic Press, New York, 2019).

6. E. A. Kuzin, N. Korneev, J. W. Haus, and B. Ibarra-Escamilla, J. Opt. Soc. Am. B 18 (2001) 919. https://doi.org/10. $1364 / \mathrm{JOSAB} .18 .000919$

7. A. Flores-Rosas et al., J. Opt. Soc. Am. B 31 (2014) 821-6. https://doi.org/10.1364/JOSAB.31.000821

8. O. Pottiez, E.A. Kuzin, B. Ibarra-Escamilla, and F. MendezMartinez, Opt. Commun. 254 (2005) 152.

9. B. Ibarra-Escamilla et al., Opt. Express 13 (2005) 10760. https://doi.org/10.1364/OPEX.13.010760

10. O. Pottiez, E.A. Kuzin, B. Ibarra-Escamilla, J.T. CamasAnzueto, and F. Gutierrez-Zainos, Electron. Lett. 40 (2004) 892.

11. O. Pottiez, E.A. Kuzin, B. Ibarra-Escamilla, J.T. CamasAnzueto, and F. Gutierrez-Zainos, Opt. Express 17 (2004) $3878 . \quad$ https://doi.org/10.1364/OPEX.12. 003878

12. O. Pottiez, B. Ibarra-Escamilla, and E.A. Kuzin, Opt. Commun. 271 (2007) 543.

13. O. Pottiez, B. Ibarra-Escamilla, and E.A. Kuzin, Opt. Express 15 (2007) 2564.

14. O. Pottiez, B. Ibarra-Escamilla, and E.A. Kuzin, Opt. Fiber Technol. 15 (2009) 258. https: / / doi .org/10.1016/j. yofte.2008.12.001

15. O. Pottiez, E. A. Kuzin, B. Ibarra-Escamilla, F. GutierrezZainos, U. Ruiz-Corona, and J. T. Camas-Anzueto, IEEE Photon. Technol. Lett. 17 (2005) 154.

16. O. Pottiez, B. Ibarra-Escamilla, and E.A. Kuzin, Opt. Commun. 281 (2008) 1037.

17. O. Pottiez, B. Ibarra-Escamilla, and E.A. Kuzin, Opt. \& Laser Technol. 41 (2009) 384-91.

18. O. Pottiez, B. Ibarra-Escamilla, and E.A. Kuzin, Opt. Fiber Technol. 15 (2009) 172.

19. A. Gonzalez-Garcia, O. Pottiez, R. Grajales-Coutiño, B. IbarraEscamilla, and E.A. Kuzin, Opt. and Laser Technol. 42 (2010) 1103.
20. O. Pottiez, B. Ibarra-Escamilla, and E.A. Kuzin, J. Europ. Opt. Soc. Rap. Public. 8 (2013) 130-59.

21. O. Pottiez et al., IEEE Photon. Technol. Lett. 27 (2015) 2272-5.

22. O. Pottiez, B. Ibarra-Escamilla, and E.A. Kuzin, Opt. Commun. 281 (2008) 982-90.

23. O. Pottiez, E.A. Kuzin, and B. Ibarra-Escamilla, IEEE Photon. Technol. Lett. 19 (2007) 1347.

24. O. Pottiez et al., Mathematical Problems in Engineering 2015 (2015) 947567.

25. O. Pottiez, R. Paez-Aguirre, J.L. Cruz, M.V. Andrés, and E.A. Kuzin, Opt. Commun. 377 (2016) 41-51. https://doi. org/10.1016/j.optcom.2016.05.029

26. E. A. Kuzin, B. Ibarra Escamilla, D. E. Garcia-Gomez, and J.W. Haus, Opt. Lett. 15 (2001) 1559-61. https://doi.org/ $10.1364 / \mathrm{OL} .26 .001559$

27. O. Pottiez, R. Grajales-Coutiño, B. Ibarra-Escamilla, E.A. Kuzin, and J.C. Hernández-García, Appl. Opt. 50 (2011) E24. https://doi.org/10.1364/AO.50.000E24

28. O. Pottiez, B. Ibarra-Escamilla, E.A. Kuzin, J.C. HernándezGarcía, A, González-García, and M. Durán-Sánchez, Laser Phys. 24 (2014) 105104.

29. H. Santiago-Hernández et al., Opt. Express 25 (2017) 25036. https://doi.org/10.1364/OE.25.025036

30. Y.E. Bracamontes-Rodríguez et al., J. Opt. 21 (2019) 045504. https://doi.org/10.1088/2040-8986/ab0bf7

31. N. Akhmediev, J.M. Soto-Crespo, and G. Town, Phys. Rev. E 63 (2001) 056602. https://doi.org/10.1103/ PhysRevE.63.056602

32. A.F.J. Runge, N.G.R. Broderick, and M. Erkintalo, Optica 2 (2015) 36-9. https://doi.org/10.1364/OPTICA.2. 000036

33. S. Chouli and P. Grelu, Opt. Express 17 (2009) 11776-81. https://doi.org/10.1364/OE.17.011776

34. F. Amrani, A. Haboucha, M. Salhi, H. Leblond, A. Komarov, and F. Sanchez, Appl. Phys. B 99 (2010) 107. https: / / doi. org/10.1007/s00340-009-3774-7

35. M. Horowitz, Y. Barad, and Y. Silberberg, Opt. Lett. 22 (1997) 799-801. https://doi.org/10.1364/OL.22. 000799

36. D.V. Churkin et al., Nat. Commun. 6 (2015) 7004. https: //doi.org/10.1038/ncomms8004

37. Z. Wang et al., Opt. Express 24 (2016) 14709.

38. S. Lee, L.A. Vazquez-Zuniga, H. Kim, Y. Kwon, K. Park, H. Kim, and Y. Jeong, Opt. Express 25 (2017) 28385-97. 
39. C. Lecaplain and Ph. Grelu, Phys. Rev. A 90 (2014) 013805. https://doi.org/10.1103/PhysRevA.90. 013805

40. A.F.J. Runge, C. Aguergaray, N.G.R. Broderick, and M. Erkintalo, Opt. Lett. 39 (2014) 319. https://doi.org/10. $1364 /$ OL.39.000319

41. S.V. Smirnov, S. Sugavanam, O.A. Gorbunov, and D.V. Churkin, Opt. Express 25 (2017) 23122-7. https://doi. org/10.1364/OE.25.023122

42. B. Li, J. Kang, S. Wang, Y. Yu, P. Feng, and K.K.Y. Wong, Opt. Lett. 44 (2019) 4351. https://doi.org/10.1364/OL. 44.004351

43. S.D. Chowdhury, B.D. Gupta, S. Chatterjee, R. Sen, and M. Pal, J. Opt. 22 (2020) 065505. https://doi.org/10.1088/ 2040-8986/ab8e9d

44. C. Lecaplain, Ph. Grelu, J.M. Soto-Crespo, and N. Akhmediev, Phys. Rev. Lett. 108 (2012) 233901. https://doi.org/ 10.1103/PhysRevLett.108.233901

45. S.D. Chowdhury, B.D. Gupta, S. Chatterjee, R. Sen, and M. Pal, Opt. Lett. 44 (2019) 2161. https://doi.org/10.1364/ OL. 44.002161

46. Y.-Q. Huang, Y.-L. Qi, Z.-C. Luo, A.-P. Luo, and W.-C. Xu, Opt. Express 24 (2016) 7356. https : / / doi .org/10. $1364 / \mathrm{OE} .24 .007356$

47. O. Pottiez, B. Ibarra-Escamilla, E.A. Kuzin, J.C. HernandezGarcia, A, Gonzalez-Garcia, and M. Duran-Sanchez, Laser Phys. 24 (2014) 015103.

48. J. P. Lauterio-Cruz et al., Opt. Express 24 (2016) 13778-87. https://doi.org/10.1364/OE.24.013778

49. A. Boucon et al., Appl. Phys. B 106 (2012) 283. https: //doi.org/10.1007/s00340-011-4816-5

50. O. Pottiez, J.C. Hernández-García, B. Ibarra-Escamilla, E.A. Kuzin, M. Duran-Sánchez, and A. Gonzalez-García, Laser Phys. 24 (2014) 115103.
51. H. Santiago-Hernández et al., Laser Phys. 25 (2015) 045106. https://doi.org/10.1088/1054-660X/25/4/ 045106

52. H. Santiago-Hernández et al., Opt. Express 23 (2015) 18840-9. https://doi.org/10.1364/OE.23.018840

53. O. Pottiez et al., Laser Phys. 28 (2018) 085108. https: //doi.org/10.1088/1555-6611/aac7e8

54. E. García-Sánchez et al., Opt. Express 24 (2016) 18917-30. https://doi.org/10.1364/OE.24.018917

55. E. García-Sánchez, O. Pottiez, Y. Bracamontes-Rodŕiguez, J.P. Lauterio-Cruz, H.E. Ibarra-Villalón, J.C. Hernández-García, M. Bello-Jiménez, and E.A. Kuzin, Laser Phys. Lett. 13 (2016) 105106.

56. J.P. Laurerio-Cruz et al., Opt. Express 27 (2019) 37196-213.

57. O. Pottiez et al., Laser Phys. Lett. 14 (2017) 105101. https: //doi.org/10.1088/1612-202x/aa8298

58. H. E. Ibarra-Villalón, O. Pottiez, Y. E. BracamontesRodríguez, J. P. Lauterio-Cruz and A. Gómez-Vieyra, Laser Phys. 28 (2018) 065103. https://doi.org/10.1088/ $1555-6611 / \mathrm{aab} 65 \mathrm{~d}$

59. Z. Wang, Z. Wang, Y.G. Liu, R. He, G. Wang, G. Yang, and S. Han, Laser Phys. Lett. 15 (2018) 055101. https: //doi.org/10.1364/OL.43.000478

60. O. Pottiez et al., Opt. Express 27 (2019) 34742-59.

61. O.S. Torres-Muñóz et al., Opt. Express 27 (2019) 17521. https://doi.org/10.1364/OE.27.017521

62. O.S. Torres-Muñóz et al., Laser Phys. 29 (2019) 115401.

63. G. Herink, F. Kurtz, B. Jalali, D. R. Solli, and C. Ropers, Science 356 (2017) 50-4. DOI: $10.1126 /$ science .aal5326

64. Z. Q. Wang, K. Nithyanandan, A. Coillet, P. Tchofo-Dinda, and Ph. Grelu, Nat. Commun. 10 (2019) 830. https://doi. org/10.1038/s41467-019-08755-4 